\title{
Atomistic Mechanisms for the Thermal Relaxation of Au-hyperdoped Si
}

\author{
W. Yang $\odot,{ }^{1,{ }^{*}, \dagger}$ Q. Hudspeth, ${ }^{2}$ P. K. Chow, ${ }^{2}$ J. M. Warrender, ${ }^{2}$ N. Ferdous, ${ }^{3}$ E. Ertekin, ${ }^{3}$ G. Malladi, ${ }^{4}$ \\ A. J. Akey, ${ }^{5}$ M. J. Aziz, ${ }^{6}$ and J. S. Williams ${ }^{1}$ \\ ${ }^{1}$ Research School of Physics and Engineering, Australian National University, Canberra, ACT 2601, Australia \\ ${ }^{2}$ U.S. Army ARDEC-Benét Laboratories, Watervliet Arsenal, New York 12189, USA \\ ${ }^{3}$ Department of Mechanical Science and Engineering and Materials Research Laboratory, University of Illinois \\ Urbana-Champaign, Urbana, Illinois 61801, USA \\ ${ }^{4}$ SUNY College of Nanoscale Science and Engineering, Albany, New York 12203, USA \\ ${ }^{5}$ Harvard University Center for Nanoscale Systems, Cambridge, Massachusetts 02138, USA \\ ${ }^{6}$ Harvard John A. Paulson School of Engineering and Applied Sciences, Cambridge, Massachusetts 02138, USA
}

(Received 19 February 2019; revised manuscript received 3 June 2019; published 8 August 2019)

\begin{abstract}
Au-hyperdoped Si produced by ion implantation and pulsed laser melting exhibits sub-band-gap absorption in the near infrared, a property that is interesting for Si photonics. However, the sub-band-gap absorption has previously been shown to be thermally metastable. In this work, we study the atomistic processes that occur during the thermal relaxation of Au-hyperdoped Si. We show that the first step in thermal relaxation is the release of substitutional Au from lattice sites. This process is characterized by an activation energy of around $1.6 \mathrm{eV}$, a value similar to that associated with $\mathrm{Au}$ diffusion in $\mathrm{Si}$, suggesting that both processes could be rate limited by the exchange of substitutional and interstitial Au atoms. As the system further relaxes, Au is found to locally diffuse and become trapped at nearby lattice defects, notably vacancies and vacancy complexes. In fact, density-functional theory results suggest that the formation of Au dimers is energetically favourable after the Au becomes locally trapped. The dimers could subsequently evolve into trimers, etc., as other diffusing Au atoms become trapped at the dimer. At low $\mathrm{Au}$ concentrations, this clustering process does not form visible precipitation after annealing at $750{ }^{\circ} \mathrm{C}$ for $3 \mathrm{~min}$. In contrast, spherical Au precipitates are found in samples with higher Au concentrations $(>0.14$ at. \%), where the $\mathrm{Au}$ atoms and the associated lattice defect distributions are laterally inhomogeneous.
\end{abstract}

DOI: 10.1103/PhysRevApplied.12.024015

\section{INTRODUCTION}

Single-crystal Si containing impurities at nonequilibrium concentrations (orders of magnitude higher than the equilibrium solid solubility limit) can be produced by ion implantation followed by pulsed laser melting (PLM) [1-3]. The ion-implanted Si layer melts during the nanosecond laser pulse and, on cooling, rapidly resolidifies into a single-crystal structure while kinetically trapping the impurities inside [4,5]. The high impurity concentrations in hyperdoped Si lead to unusual optoelectronic properties that are of wide interest. With conventional dopants such as $\mathrm{B}, \mathrm{P}, \mathrm{As}$, and $\mathrm{Sb}$, the ultraheavily doped layers give rise to correspondingly high electrical activity [6,7]. When impurities such as chalcogens and

\footnotetext{
*winney007@gmail.com

$\dagger$ Present address: Research School of Electrical, Energy and Material Engineering, Australian National University, Canberra, ACT 2601, Australia.
}

suitable transition metals are used to hyperdope $\mathrm{Si}$, the discrete sub-band-gap levels that they introduce extend into an intermediate band, enabling room-temperature subband-gap photoresponse at near- to mid-IR wavelengths [8-10], a behavior which is significant for Si photonics $[11,12]$. Novel behaviors have also been demonstrated in hyperdoped $\mathrm{Si}$ nanostructures such as nanocrystals and nanowires $[13,14]$.

Given the highly nonequilibrium impurity concentrations achieved, the metastability of hyperdoped Si under subsequent thermal annealing is hardly surprising. During thermal annealing, Si hyperdoped with As, for example, has been shown to form electrically inactive clusters and As-vacancy complexes (which appear to be substitutional in the Si lattice), which then evolve into precipitates $[15,16]$. A similar behavior has also been observed with Sb-hyperdoped Si and As-hyperdoped Ge [17-19]. In Si hyperdoped with chalcogens ( $\mathrm{S}, \mathrm{Se}$, and $\mathrm{Te}$ ), a decrease in sub-band-gap absorption after thermal annealing has been observed, with thermally activated formation 
of electrically inactive dopant configurations being identified as a relevant mechanism in the deactivation process $[20,21]$. In addition, kinetically limited processes have also been shown to contribute to the deactivation of the sub-band-gap optical absorption [22]. Such processes are characterized by a lack of long-range impurity diffusion, short-range trapping, and possible precipitation [20,21,23], and have been hypothesized to give rise to chemical states that are optically inactive [21].

With energy levels deeper in the band gap of $\mathrm{Si}$ than chalcogens, transition metals have also gained considerable interest as candidates for hyperdoping Si. For instance, a near-IR photodetector fabricated from Au-hyperdoped Si has already been demonstrated [9]. Clearly, further device development necessitates an understanding of the subsequent thermal stability of the hyperdoped material, especially since the formation of contacts and other device structures, such as antireflective coatings, requires elevated-temperature processing following PLM. Although the annealing behavior of Si hyperdoped with conventional dopants and chalcogens is relatively well studied, similar studies on $\mathrm{Si}$ hyperdoped with transition metals remain limited to $\mathrm{Ti}$ [24] and $\mathrm{Au}$ [25]. Studies on Ti-hyperdoped Si have focused only on the changes in electrical properties after annealing, and no structural characterization has been attempted. However, it is worth noting that the formation of $\mathrm{Ti}$ silicide has been hypothesized to be responsible for the changes in electrical behavior [24]. In Au-hyperdoped $\mathrm{Si}$, we have previously reported an annealing-induced decrease in sub-band-gap absorption that can be correlated with a reduction in Au substitutionality, as Au was found to move off lattice sites during annealing [25]. Nevertheless, the details of this process were not investigated and the study focused only on samples with a high Au concentration ( $>1$ at. \%). Furthermore, the atomistic behavior of the supersaturated Au atoms during thermal annealing was not specifically examined. To this end, the current paper aims to provide a systematic study of the behavior of Au during the thermal relaxation of hyperdoped $\mathrm{Si}$. Because Au is a well-studied impurity in $\mathrm{Si}$ at least at equilibrium concentrations, there is a wellestablished framework for understanding its behavior in hyperdoped Si. In particular, we show that the first step in this multistep process is controlled by the same activation energy for all Au concentrations studied, and is also similar to that previously found for the diffusion of $\mathrm{Au}$ in $\mathrm{Si}$.

In this work, Au-hyperdoped Si produced by ion implantation and PLM is subjected to isochronal rapid thermal annealing (RTA). We have chosen to use RTA to carry out this study because the fast ramping rates associated with RTA compared with furnace annealing will result in less $\mathrm{Au}$ diffusion during ramping. This not only is more industrially relevant (it is desirable to retain the high $\mathrm{Au}$ concentrations during post-PLM processes) but will also allow us to study the thermal behavior more accurately. The change in Au distribution and atom location at different temperatures is monitored by Rutherford backscattering spectrometry combined with channeling (RBS-C), and the structural changes are examined by cross-sectional transmission electron microscopy (XTEM) for selected samples. We examine the role of Au diffusion and trapping in the thermal relaxation process and explore the possibility of $\mathrm{Au}$ dimerization during the thermal relaxation by comparing experimental results with first-principles calculations by density-functional theory (DFT) published previously in Ref. [26], to which the reader is referred for a more comprehensive analysis of the DFT data. In addition, we explore whether the relaxation behavior depends on the $\mathrm{Au}$ concentration. This is especially relevant as two distinct Au concentration regimes were previously identified for $\mathrm{Au}$ in hyperdoped Si formed by rapid solidification: a homogeneous regime at low $\mathrm{Au}$ concentrations, where the $\mathrm{Au}$ distribution is laterally uniform, and an inhomogeneous regime at high $\mathrm{Au}$ concentrations (greater than approximately 0.2 at. \%), where Au-rich filaments are observed [25].

\section{EXPERIMENTAL METHODS}

Samples are prepared by implanting various doses of $\mathrm{Au}^{+}$into (100)-oriented $n$-type $\mathrm{Si}$ wafers (double-side polished, phosphorus-doped, resistivity $1-10 \Omega \mathrm{cm}$ ) at energies ranging from 50 to $300 \mathrm{keV}$ at liquid-nitrogen temperature in vacuum. Two samples (implanted at 200 and $300 \mathrm{keV}$ ) are analyzed in detail in this paper, as the $\mathrm{Au}$ concentrations achieved in these samples are representative of the homogeneous and inhomogeneous cases, respectively. The implanted samples are subsequently melted by a single laser pulse from a Nd:YAG laser. The sample that receives a $200-\mathrm{keV}$ implant with a Au dose of $1 \times 10^{15} \mathrm{~cm}^{-2}$ has a peak subsurface $\mathrm{Au}$ concentration of 0.14 at. \% (as measured by RBS-C); this sample is described as the low-concentration sample in our analysis below. The sample that receives a $300-\mathrm{keV}$ implant with a $\mathrm{Au}$ dose of $6 \times 10^{15} \mathrm{~cm}^{-2}$ has a peak subsurface $\mathrm{Au}$ concentration of 1.4 at. \% and is described as the high-concentration sample. A detailed description of the ion implantation and PLM conditions can be found in Ref. [25].

Rapid thermal annealing is performed in a flowing Ar ambient using a JetFirst RTA furnace. The temperature profiles are recorded by a thermocouple attached to the back of a virgin Si wafer onto which the samples are placed. The nominal annealing temperature is varied between $350^{\circ} \mathrm{C}$ and $850^{\circ} \mathrm{C}$, and the annealing duration is kept constant at $3 \mathrm{~min}$. RBS and channeling measurements are performed using $2-\mathrm{MeV} \mathrm{He}^{+}$with 
the RBS system at the Australian National University. A glancing-angle detector situated $78^{\circ}$ from the sample surface normal is used for optimum depth resolution [27]. TEM is performed using a JEOL $2100 \mathrm{~F}$ microscope on cross-sectional samples prepared using conventional ion-polishing.

\section{RESULTS AND DISCUSSION}

\section{A. Change in Au profile}

Figures 1(a) and 1(b) show the Au distribution (obtained from RBS) as a function of annealing temperature for the low- and high-concentration samples, respectively. For annealing temperatures up to $750^{\circ} \mathrm{C}$, the Au distribution in the low-concentration sample remains unchanged. While the $\mathrm{Au}$ distribution in the high-concentration sample also shows no obvious change for annealing temperatures at or below $550^{\circ} \mathrm{C}$ [shown in Fig. 1(b) inset], the sharp concentration peak at approximately $120 \mathrm{~nm}$ below the surface begins to flatten and shift toward the surface at temperatures above $550{ }^{\circ} \mathrm{C}$, indicating outward Au migration in the top $120 \mathrm{~nm}$ of the sample. At the highest annealing temperature studied $\left(850^{\circ} \mathrm{C}\right)$, the peak Au concentration drops to approximately 0.9 at. $\%$ and the profile is flattened in the top approximately $65-120 \mathrm{~nm}$. XTEM images of both samples are shown above the respective RBS spectra. Consistent with previous reports on samples fabricated under similar conditions [25], no visible defects are observed in the low-concentration sample, while filamentary features are observed in the high-concentration case. Note that the surface Au content decreases marginally with annealing temperature in both cases, presumably because some $\mathrm{Au}$ evaporates during the annealing process, a behavior which has been observed before [28].
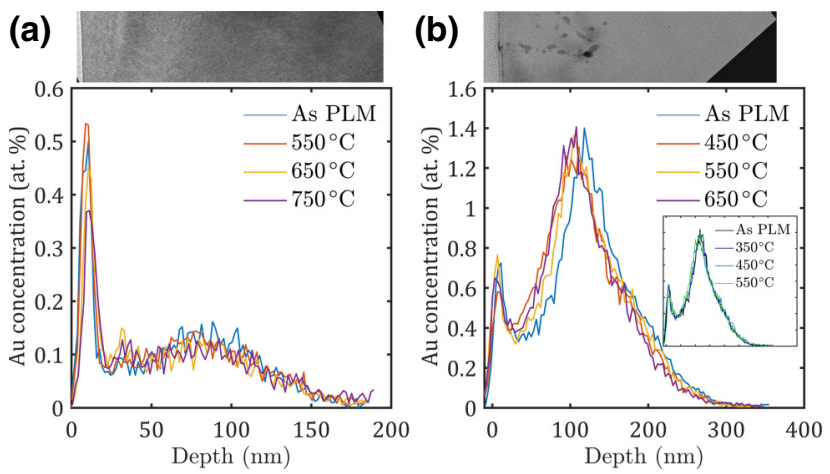

FIG. 1. (a) Change in the Au concentration profile for (a) the low-concentration sample and (b) the high-concentration sample after various isochronal anneals, as measured by RBS, plotted against XTEM images of the corresponding as-PLM samples (before annealing) with the same depth scale; the inset shows that the Au concentration profiles virtually overlap for annealing temperatures below $550^{\circ} \mathrm{C}$. The scale bars drawn on the XTEM images are $50 \mathrm{~nm}$.
The $\mathrm{Au}$ concentration in the hyperdoped layer exceeds the equilibrium solubility by orders of magnitude, even in the low-concentration case. Therefore, at the annealing temperatures studied here, the supersaturated $\mathrm{Au}$ is expected to relax toward equilibrium by thermally diffusing down the concentration gradient at or below the equilibrium solubility limit into the Au-free Si bulk, and to precipitate at favorable sites [29], as will be discussed in more detail later. This would cause a change in the Au profile, which is in fact observed only for the highconcentration sample, as shown in Fig. 1(b). Thus, the lack of change in the Au profile for the low-concentration sample [Fig. 1(a)] is rather surprising. As such, we next investigate the change in the lattice position of the $\mathrm{Au}$ atoms by RBS-C.

\section{B. Change in Au lattice position}

The change in the Au substitutionality can be deduced from RBS-C spectra as a function of annealing temperature. Here, the Au substitutionality is calculated by comparing the $\mathrm{Au}$ backscatter yield from a direction that is aligned with a crystallographic axis ("channeled") and from a random direction ("random") [27]. Figures 2(a) and (a)

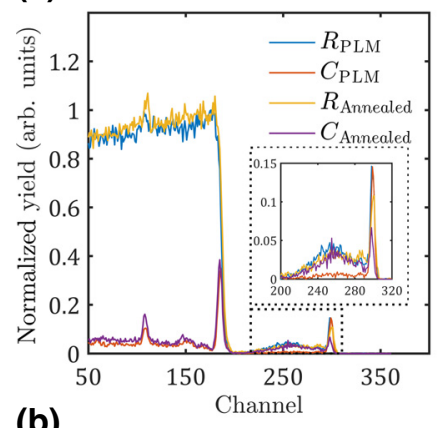

(b)

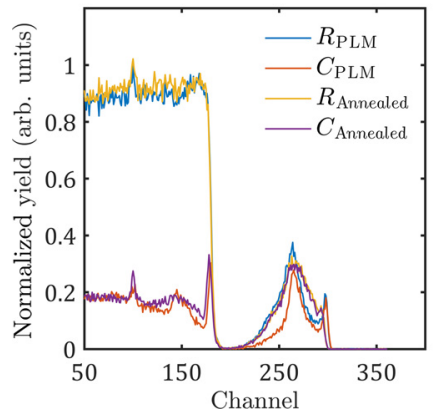

(c)

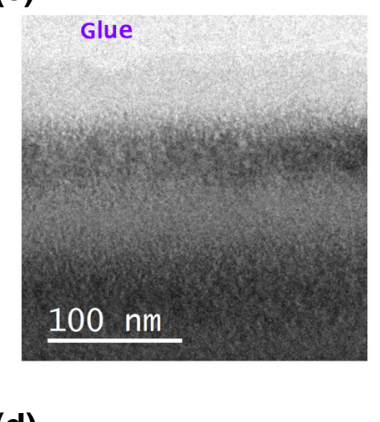

(d)

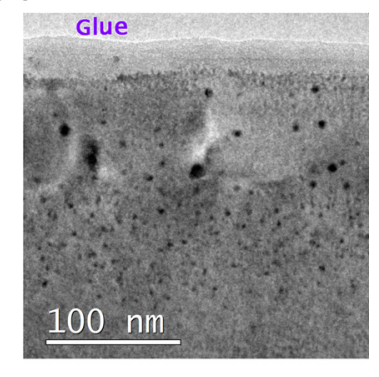

FIG. 2. (a) Random $(R)$ and channeled (C) RBS-C spectra for the low-concentration sample, before and after RTA at $750^{\circ} \mathrm{C}$ for $3 \mathrm{~min}$. The inset is a magnified view of the Au region of the spectra from channel 200 to channel 320. (b) Random $(R)$ and channeled $(C)$ RBS-C spectra for the high-concentration sample, before and after RTA under the same conditions as for (a). XTEM images taken after RTA at $750^{\circ} \mathrm{C}$ for 3 min of (c) the low-concentration sample and (d) the high-concentration sample. 
2(b) compare backscattering spectra (one taken from a random direction and one taken from the channeled direction) of both the low-concentration and the high-concentration sample before and after a $750{ }^{\circ} \mathrm{C}$ anneal for $3 \mathrm{~min}$.

Both samples exhibit a drastic decrease in Au substitutionality after annealing. However, examination of these samples by XTEM, as shown in Figs. 2(c) and 2(d), reveals that the loss of $\mathrm{Au}$ substitutionality leads to observable $\mathrm{Au}$ precipitation in the high-concentration sample but not in the low-concentration sample. In fact, the XTEM image of the low-concentration sample after annealing [Fig. 2(c)] appears to be identical to that taken before annealing [Fig. 1(a)], with no signs of Au clustering or precipitation at the resolution of XTEM. As we demonstrate later, the apparent difference in the precipitation behavior between the two samples is related to the trapping of $\mathrm{Au}$ atoms to local sinks.

\section{The thermal relaxation process}

The mechanism by which the $\mathrm{Au}$ substitutionality decreases is further investigated by monitoring of the rate of substitutionality loss as a function of annealing temperature. Before RTA, and excluding the segregated $\mathrm{Au}$ on the surface, the average substitutional $\mathrm{Au}$ fractions $\left(X_{\text {PLM }}\right)$ within the hyperdoped layer are $87 \%$ and $50 \%$ for the low- and high-concentration samples, respectively. This result is consistent with substitutionality values obtained previously for similar $\mathrm{Au}$ concentrations [25], although the measured substitutional fraction for the high-concentration sample may be lower than the actual value due to lattice disorder (strain) around the inhomogeneous $\mathrm{Au}$ filaments. In both samples, this $\mathrm{Au}$ substitutionality remains roughly unchanged for annealing temperatures lower than $400^{\circ} \mathrm{C}$. At higher temperatures, the $\mathrm{Au}$ substitutionality decreases monotonically with the annealing temperature. Although not shown here, we observed the same qualitative behavior for a range of Au-hyperdoped samples with implant energies ranging from 50 to $300 \mathrm{keV}$, and doses ranging from $7 \times 10^{14}$ to $6 \times 10^{15} \mathrm{~cm}^{-2}$.

To quantify the change in substitutional Au loss, we normalize the change in Au substitutionality, $\Delta X$, to the substitutionality of the sample measured directly after PLM (i.e., with no subsequent annealing) using

$$
\Delta X=\frac{X_{\mathrm{PLM}}-X(T)}{X_{\mathrm{PLM}}}
$$

where $X_{\mathrm{PLM}}$ is the substitutional Au fraction in the pulsedlaser-melted sample (with no anneal) and $X(T)$ is that after subsequent annealing at temperature $T$.

In Fig. 3, the natural logarithm of $\Delta X / t$ (i.e., the average rate of substitutionality loss) is plotted against $1 / T$ for both samples. For the linear regions on the right (annealing temperature $<500^{\circ} \mathrm{C}$ ), the average rate at which the

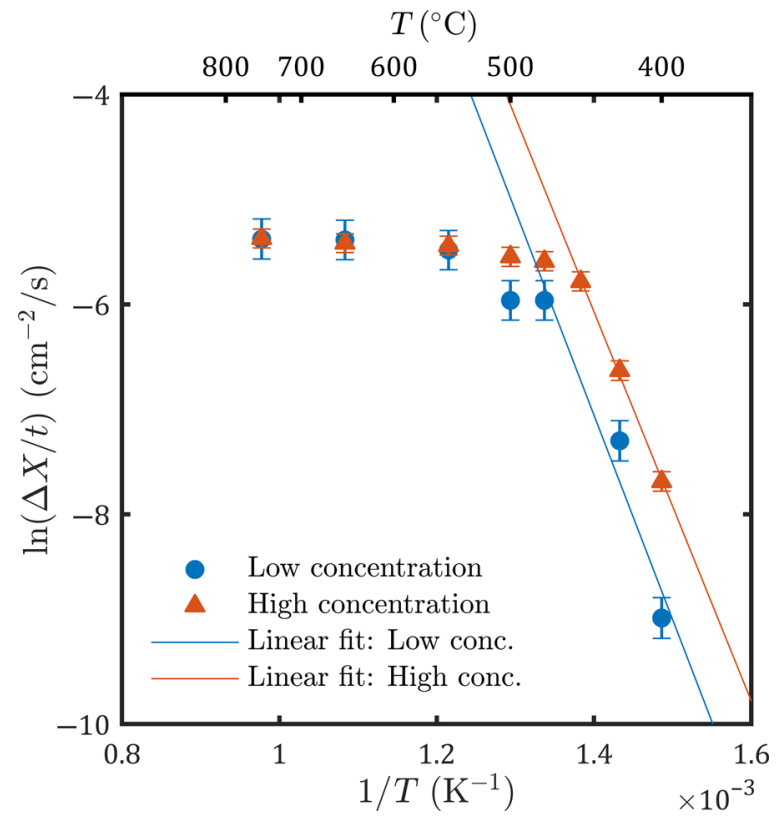

FIG. 3. Arrhenius plot of the loss of substitutionality for both low and high $\mathrm{Au}$ concentrations. Linear fits are obtained by total least-squares fitting using the York error-in-variable model [30] for $T<500{ }^{\circ} \mathrm{C}$.

$\mathrm{Au}$ substitutionality decreases can be described by the Arrhenius equation,

$$
\frac{\Delta X}{t}=A e^{E_{a} / k T}
$$

where $t$ is the annealing duration, $A$ is a rate constant, $E_{a}$ is the activation energy for the process, and $k$ is the Boltzmann constant. In Fig. 3, an annealing duration $t=180 \mathrm{~s}$ is used for all annealing temperatures and we assume that the rate of substitutionality loss is constant during this time [31]. Thus, the activation energy for the loss in $\mathrm{Au}$ substitutionality is reasonably approximated by the slope of the straight line, where activation energies of $E_{a}=1.6 \pm 0.1 \mathrm{eV}$ and $1.7 \pm 0.2 \mathrm{eV}$ can be extracted from the data for the low- and high-concentration samples, respectively. The activation energy extracted from samples with other Au concentrations, as tabulated in Table I, is also found to be consistent within experimental error. Thus, the process that is characterized by this activation energy for the initial change in Au substitutionality appears to be the same for all $\mathrm{Au}$ concentrations studied here. At annealing temperatures above $500^{\circ} \mathrm{C}$, the curves shown in Fig. 3 become nonlinear and can no longer be characterized by the same activation energy. This suggests that additional thermally activated processes are occurring concurrently with the loss of substitutionality in this temperature regime $\left(T>500^{\circ} \mathrm{C}\right)$.

It is interesting to note that the activation energy extracted for the loss of substitutional $\mathrm{Au}$ in hyperdoped 
TABLE I. Activation energies $\left(E_{a}\right)$ extracted from various Au-hyperdoped samples after a 3 min isochronal rapid thermal anneal at annealing temperatures $<450{ }^{\circ} \mathrm{C}$. $[\mathrm{Au}]_{P}$ is the peak subsurface Au concentration, and $X_{\mathrm{PLM}}$ is the subsurface substitutional Au fraction.

\begin{tabular}{cccccc}
\hline \hline Implant energy $(\mathrm{keV})$ & Dose $\left(\mathrm{cm}^{-2}\right)$ & {$[\mathrm{Au}]_{P}($ at. $\%)$} & $X_{\mathrm{PLM}}$ & Inhomogeneous? & $E_{a}(\mathrm{eV})$ \\
\hline 50 & $7 \times 10^{14}$ & 0.09 & $87 \%$ & No & $1.5 \pm 0.3$ \\
50 & $1 \times 10^{15}$ & 0.18 & $75 \%$ & No & $1.4 \pm 0.2$ \\
200 & $1 \times 10^{15}$ & 0.10 & $87 \%$ & No & $1.6 \pm 0.1$ \\
200 & $4 \times 10^{15}$ & 0.44 & $67 \%$ & Yes & $1.5 \pm 0.2$ \\
300 & $6 \times 10^{15}$ & 1.4 & $50 \%$ & Yes & $1.7 \pm 0.2$ \\
\hline \hline
\end{tabular}

$\mathrm{Si}$ (approximately $1.6 \mathrm{eV}$ ) is similar to the activation energy (approximately $1.7 \pm 0.1 \mathrm{eV}$ ) for the diffusion of $\mathrm{Au}$ extracted from near-equilibrium annealing experiments on Au-implanted Si [32]. One plausible interpretation is that both processes are controlled by the same thermally activated step, which we discuss below in relation to the diffusion of $\mathrm{Au}$ in $\mathrm{Si}$.

Two indirect substitutional-interstitial mechanisms have been proposed to explain the diffusion behavior of $\mathrm{Au}$ in $\mathrm{Si}$. In the kick-out mechanism [33], a diffusing interstitial Au atom "kicks out" a Si atom in the lattice, takes its place, and creates a Si self-interstitial. This is described by

$$
\mathrm{Au}_{i} \leftrightarrow \mathrm{Au}_{s}+I
$$

where $I$ denotes a Si self-interstitial. Analogously, the dissociative or Frank-Turnbull mechanism, which was originally proposed for $\mathrm{Cu}$ diffusion in $\mathrm{Ge}$ [34], has subsequently been suggested for Au diffusion in Si [35]: it involves the production of a vacancy, $V$. In this scenario, $\mathrm{Au}_{s}$ is dissociated into a $\mathrm{Au}_{i}$ and a $V$. This process is described by

$$
\mathrm{Au}_{s} \leftrightarrow \mathrm{Au}_{i}+V
$$

Although there is a preponderance of evidence for the kickout mechanism, the literature does not show unanimous agreement (see, for instance, Refs. [36,37] and references within). However, it has been demonstrated that when the sample contains a supersaturation of sinks for diffusing $I$ or $V$ [i.e., when $I$ in Eq. (3) and $V$ in Eq. (4) are removed sufficiently quickly via recombination and do not limit the diffusion], the diffusion profiles for the two mechanisms become identical and the exchange of substitutional and interstitial $\mathrm{Au}$ becomes the rate-limiting process instead [38]. This latter exchange-limited regime is likely to be operative during the diffusion of ion-implanted $\mathrm{Au}$, from which an activation energy of approximately $1.7 \pm 0.1 \mathrm{eV}$ was extracted in Ref. [32], because ion-implanted Si is known to contain sinks for point defects. For instance, the so-called $R_{P} / 2$ defects, which are excess vacancies created by the implantation process, are found to cluster into thermally stable small voids in ion-implanted and thermally annealed Si [39].
Furthermore, the release of $\mathrm{Au}$ from substitutional lattice positions can be written as

$$
\mathrm{Au}_{s} \rightarrow \mathrm{Au}_{i}+V
$$

where $V$ denotes a vacancy. In this case, the exchange process between $\mathrm{Au}_{s}$ and $\mathrm{Au}_{i}$ would also be the ratelimiting process assuming (a) the migration of $\mathrm{Au}_{i}$ and (b) further creation of vacancies do not limit the reaction. Such assumptions are not unreasonable, because (a) $\mathrm{Au}_{i}$ is known to have an extremely high diffusivity in Si [40], and (b) excess vacancies in Si are known to cluster at favorable sites, as mentioned earlier [39]. While the analysis above strongly suggests that the release of $\mathrm{Au}$ from substitutional sites is the rate-limiting step (characterized by an $E_{a}$ of around $1.6 \mathrm{eV}$ ) for both the thermal diffusion of ion-implanted $\mathrm{Au}$ in $\mathrm{Si}$ and the loss of Au substitutionality in Au-hyperdoped Si, further work is required to fully validate this interpretation. Regardless of the physical origin of the extracted $E_{a}$, however, the $E_{a}$ value of approximately $1.6 \mathrm{eV}$ is consistent for all $\mathrm{Au}$ concentrations studied [41]. However, the apparent difference in the final relaxation behavior (precipitation) between the lowand high-concentration samples (as shown in Fig. 1) now needs to be addressed.

It is well known that lattice imperfections such as point defects, cavities, and free surfaces are effective sinks for metal impurities in $\mathrm{Si}$ [42]. In particular, atomic trapping has been previously observed for equilibriumconcentration $\mathrm{Au}$ in $\mathrm{Si}$, during which $\mathrm{Au}$ atoms are found to be trapped on cavity walls through a chemisorptionlike process. Similarly, other imperfect regions in the lattice (such as dislocations and other defects) are also likely to be active trapping sites as a result of poorly coordinated Si atoms in or around such regions. Such local lattice disorder and excess vacancies are expected to trap diffusing $\mathrm{Au}_{i}$ atoms. Although the hyperdoped layer is near-perfect crystalline $\mathrm{Si}$ (as seen in high-resolution TEM [25]), in a separate study [43], we demonstrated that it does contain excess vacancies and is hence not defectfree. Because of the presence of such traps, the excess $\mathrm{Au}$ (above the equilibrium solubility limit) that was initially frozen into near-substitutional lattice sites following PLM would, after being released from substitutional sites, 
diffuse to favorable local trapping sites within the hyperdoped layer. While this behavior is expected at both $\mathrm{Au}$ concentrations studied, we believe that the differing size, distribution, and binding energy of the trap sites between the two concentration regimes is the cause of the apparent difference in precipitation behavior seen in Fig. 2.

Unlike the trapping effect observed in equilibriumconcentration ion-implanted samples during thermal annealing, where long-range diffusion of $\mathrm{Au}$ to cavities or the wafer surfaces occurs $[29,42]$, the trapping sites in Au-hyperdoped Si (for example, vacancies) are confined to the hyperdoped layer (i.e., within the PLM melt depth). Hence, the migration and trapping of the $\mathrm{Au}$, for both $\mathrm{Au}$ concentrations, are also expected to be local within the hyperdoped layer. For the high-concentration case, the most favorable trap sites (i.e., with the highest binding energy) are presumably concentrated within the top approximately $110 \mathrm{~nm}$, in which Au-rich filaments and a variety of associated lattice defects can be observed [see Fig. 1(b)]. As the annealing temperature increases further, the trap sites with the highest binding energy (such as vacancies and small cavities [29]) may then cluster and become nucleation sites for subsequent $\mathrm{Au}$ precipitation and growth, resulting in skewing and broadening of the $\mathrm{Au}$ profile. Conversely, the lack of profile broadening observed in the low-concentration sample on annealing [Fig. 1(a)] suggests very local Au trapping where the migration length is within the resolution of RBS. The homogeneity observed in TEM in Fig. 1(a), in contrast to the high-concentration sample shown in Fig. 1(b), suggests a more uniform concentration of trapping sites interspersed within the melt depth at sufficiently high density that none of them attracts enough $\mathrm{Au}$ atoms to form a visible precipitate. Rather, we suggest that the multitudinous trapping sites, all of which are small compared with the sinks in the high-concentration case, give rise to a greater number of much smaller Au complexes that pin Au sufficiently strongly to prevent the formation of visible precipitates at temperatures up to $750^{\circ} \mathrm{C}$.

Although previous high-resolution TEM indicated the formation of spherical precipitates as the final stage of annealing [25], experimental observation of the atomistic evolution of this process is difficult even with high-resolution TEM, considering that the transient configurations of the trapped Au may consist of only up to a few $\mathrm{Au}$ atoms. However, a first-principles analysis by DFT has been recently employed to simulate the defect formation energetics for different atomic configurations of supersaturated Au in Si [26]. Since DFT gives the equilibrium configuration when all the atoms are relaxed, the simulated system can give insight into the relaxation process of the Au-hyperdoped Si system following subsequent thermal annealing. We now use these first-principles DFT results to aid the understanding of the atomistic behavior of Au-hyperdoped Si during thermal relaxation.
During the initial stage of relaxation, the loss of $\mathrm{Au}$ substitutionality observed in RBS measurements is ratelimited by the release of $\mathrm{Au}$ from substitutional lattice sites and subsequent local migration to adjacent trapping sites such as lattice disorder and, especially, vacancy-type defects [43]. Because the substitutional Au dimer configuration is found to be the most energetically favorable defect reported in Ref. [26], the Au that is released from isolated substitutional sites will strongly favor the formation of a dimer, after the atoms become locally trapped (at isolated vacancies, for example) and relax in adjacent sites. Other migrating $\mathrm{Au}$ atoms in proximity to the dimer complex are expected to be attracted to the dimer in a similar fashion to lower the free energy [44], evolving into trimers and so on, ultimately leading to the nucleation of observable precipitates. Compared with the low-concentration sample, the trap sites in the high-concentration sample are inhomogeneous and larger, which may result in larger Audecorated zones that can nucleate and grow into larger, well-defined $\mathrm{Au}$ precipitates. This process is consistent with the continual broadening of the Au concentration profile with increasing annealing temperature for $T>500^{\circ} \mathrm{C}$, as shown in Fig. 1(b).

Finally, DFT results indicate that different $\mathrm{Au}$ configurations in the Si lattice are associated with different defect levels within the Si band gap. In particular, isolated substitutional $\mathrm{Au}$, which is experimentally found to be the primary $\mathrm{Au}$ configuration in an unrelaxed system, is expected to give rise to optically active midgap

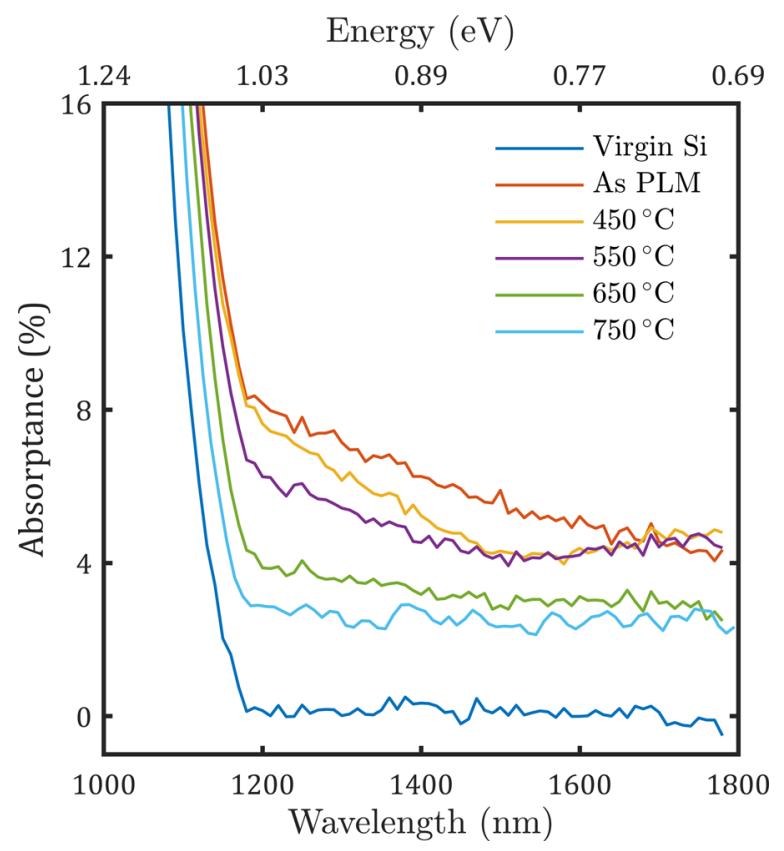

FIG. 4. Sub-band-gap absorption of the low-concentration sample after 3-min anneals at temperatures ranging from $450{ }^{\circ} \mathrm{C}$ to $750{ }^{\circ} \mathrm{C}$. The absorption of the high-concentration sample has been reported elsewhere [25]. 
states that are responsible for the observed sub-band-gap optical absorption [26]. Au dimers, on the other hand, are not expected to introduce such midgap states based on DFT calculations. This result is qualitatively consistent with the reduction in sub-band-gap optical absorption observed in annealed samples with a high Au concentration, as reported previously [25]. Similarly, as is shown in Fig. 4, the sub-band-gap optical absorption for the low-concentration sample studied here also decreases with annealing temperature. The upward tail from approximately $1500 \mathrm{~nm}$ that is observed following the $450{ }^{\circ} \mathrm{C}$ and $550^{\circ} \mathrm{C}$ anneals has also been observed previously in the high-concentration sample, and is hypothesized to be related to other, non-Au-related defects in the Si lattice that anneal away at temperatures higher than $550^{\circ} \mathrm{C}$. Note that the sub-band-gap absorption never drops to the virgin level even after a $750{ }^{\circ} \mathrm{C}$ anneal. As discussed previously [25], this is a result of a small fraction of the Au diffusing throughout the $450-\mu \mathrm{m}$-thick wafer and subsequently occupying substitutional sites up to the equilibrium solubility limit throughout the sample at $750{ }^{\circ} \mathrm{C}$ (approximately $\left.1 \times 10^{14} \mathrm{~cm}^{-3}\right)$.

\section{CONCLUSION}

In conclusion, we have shown that the thermal relaxation of Au-hyperdoped $\mathrm{Si}$ is a multistep process. The first step of relaxation is marked by a loss in the Au substitutionality through a process that is characterized by an activation energy of approximately $1.6 \mathrm{eV}$, a value that may be related to the rate-limiting step in the thermal diffusion of $\mathrm{Au}$ in $\mathrm{Si}$. This suggests that the rate-limiting step in both cases may be the exchange between substitutional and interstitial Au. As the relaxation progresses, our observations of the Au profile broadening and local lattice environment strongly suggest local Au trapping at lattice imperfections, such as vacancies, within the hyperdoped layer. The trapped Au could then evolve into the more energetically favorable configurations of Au dimers and trimers. The system becomes fully relaxed when all the excess $\mathrm{Au}$ atoms that are initially incorporated during solidification into substitutional lattice sites beyond the solubility limit evolve into observable Au precipitates (at high concentrations). Although these observations are conclusive only for $\mathrm{Au}$-hyperdoped $\mathrm{Si}$, it is conceivable that similar thermal behaviors could also take place in other hyperdoped materials.

\section{ACKNOWLEDGMENTS}

Funding from the U.S. Army under Contract No. FA5209-16-P-0104 is acknowledged for partial support of this work. We acknowledge access to NCRIS facilities (ANFF and the Heavy Ion Accelerator Capability) and the Centre of Advanced Microscopy at the Australian National University. We thank Dr. P. Kremer for assistance with the
TEM. This work was performed in part at the Center for Nanoscale Systems (CNS), a member of the National Nanotechnology Coordinated Infrastructure Network (NNCI), which is supported by the National Science Foundation under NSF Grant No. 1541959. CNS is part of Harvard University.

[1] Jeffrey M. Warrender, Laser hyperdoping silicon for enhanced infrared optoelectronic properties, Appl. Phys. Rev. 3, 031104 (2016).

[2] Michael J. Aziz, Interface attachment kinetics in alloy solidification, Metall. Mater. Trans. A 27A, 671 (1996).

[3] J. M. Poate and James W. Mayer, eds., Laser Annealing of Semiconductors (Academic Press, New York, 1982).

[4] S. U. Campisano, G. Foti, P. Baeri, M. G. Grimaldi, and E. Rimini, Solute trapping by moving interface in ionimplanted silicon, Appl. Phys. Lett. 37, 719 (1980).

[5] M. J. Aziz, Model for solute redistribution during rapid solidification, J. Appl. Phys. 53, 1158 (1982).

[6] N. Natsuaki, M. Tamura, and T. Tokuyama, Nonequilibrium solid solutions obtained by heavy ion implantation and laser annealing, J. Appl. Phys. 51, 3373 (1980).

[7] P. M. Rousseau, P. B. Griffin, W. T. Fang, and J. D. Plummer, Arsenic deactivation enhanced diffusion: A time, temperature, and concentration study, J. Appl. Phys. 84, 3593 (1998).

[8] Brion P. Bob, Atsushi Kohno, Supakit Charnvanichborikarn, Jeffrey M. Warrender, Ikurou Umezu, Malek Tabbal, James S. Williams, and Michael J. Aziz, Fabrication and subband gap optical properties of silicon supersaturated with chalcogens by ion implantation and pulsed laser melting, J. Appl. Phys. 107, 123506 (2010).

[9] J. P. Mailoa, A. J. Akey, C. B. Simmons, D. Hutchinson, J. Mathews, J. T. Sullivan, D. Recht, M. T. Winkler, J. S. Williams, J. M. Warrender, P. D. Persans, M. J. Aziz, and T. Buonassisi, Room-temperature sub-band gap optoelectronic response of hyperdoped silicon, Nat. Commun. 5, 3011 (2014).

[10] K. Sánchez, I. Aguilera, P. Palacios, and P. Wahnón, Formation of a reliable intermediate band in Si heavily coimplanted with chalcogens (S, Se, Te) and group III elements (B, Al), Phys. Rev. B 82, 165201 (2010).

[11] Maurizio Casalino, Giuseppe Coppola, Mario Iodice, Ivo Rendina, and Luigi Sirleto, Near-infrared sub-bandgap allsilicon photodetectors: State of the art and perspectives, Sensors 10, 10571 (2010).

[12] Richard Soref, Mid-infrared photonics in silicon and germanium, Nat. Photonics 4, 495 (2010).

[13] Shu Zhou, Xiaodong Pi, Zhenyi Ni, Qingbin Luan, Yingying Jiang, Chuanhong Jin, Tomohiro Nozaki, and Deren Yang, Boron- and phosphorus-hyperdoped silicon nanocrystals, Part. Part. Syst. Char. 32, 213 (2015).

[14] Nipun Misra, Li Xu, Yaoling Pan, Nathan Cheung, and Costas P. Grigoropoulos, Excimer laser annealing of silicon nanowires, Appl. Phys. Lett. 90, 111111 (2007).

[15] J. L. Allain, J. R. Regnard, A. Bourret, A. Parisini, A. Armigliato, G. Tourillon, and S. Pizzini, Extended $\mathrm{X}$-ray-absorption fine-structure study of the local atomic 
structure in As+ heavily implanted silicon, Phys. Rev. B 46, 9434 (1992).

[16] D. Christoph Mueller, Eduardo Alonso, and Wolfgang Fichtner, Arsenic deactivation in $\mathrm{Si}$ : Electronic structure and charge states of vacancy-impurity clusters, Phys. Rev. B 68, 045208 (2003).

[17] Yayoi Takamura, Arturas Vailionis, Ann F. Marshall, Peter B. Griffin, and James D. Plummer, Dopant deactivation in heavily Sb doped Si (001): A high-resolution X-ray diffraction and transmission electron microscopy study, J. Appl. Phys. 92, 5503 (2002).

[18] J. S. Williams and K. T. Short, Metastable doping behavior in antimony-implanted (100) silicon, J. Appl. Phys. 53, 8663 (1982).

[19] R. Milazzo, G. Impellizzeri, D. Piccinotti, D. De Salvador, A. Portavoce, A. La Magna, G. Fortunato, D. Mangelinck, V. Privitera, A. Carnera, and E. Napolitani, Low temperature deactivation of Ge heavily n-type doped by ion implantation and laser thermal annealing, Appl. Phys. Lett. 110, 011905 (2017).

[20] Brian R. Tull, Mark T. Winkler, and Eric Mazur, The role of diffusion in broadband infrared absorption in chalcogendoped silicon, Appl. Phys. A: Mater. Sci. Process. 96, 327 (2009).

[21] C. B. Simmons, Austin J. Akey, Jacob J. Krich, Joseph T. Sullivan, Daniel Recht, Michael J. Aziz, and Tonio Buonassisi, Deactivation of metastable single-crystal silicon hyperdoped with sulfur, J. Appl. Phys. 114, 243514 (2013).

[22] Bonna K. Newman, Meng-Ju Sher, Eric Mazur, and Tonio Buonassisi, Reactivation of sub-bandgap absorption in chalcogen-hyperdoped silicon, Appl. Phys. Lett. 98, 251905 (2011).

[23] T. G. Kim, Jeffrey M. Warrender, and Michael J. Aziz, Strong sub-band-gap infrared absorption in silicon supersaturated with sulfur, Appl. Phys. Lett. 88, 241902 (2006).

[24] J. Olea, D. Pastor, I. Mártil, and G. González-Díaz, Thermal stability of intermediate band behavior in $\mathrm{Ti}$ implanted Si, Sol. Energy Mater. Sol. Cells 94, 1907 (2010).

[25] W. Yang, A. J. Akey, L. A. Smillie, J. P. Mailoa, B. C. Johnson, J. C. McCallum, D. Macdonald, T. Buonassisi, M. J. Aziz, and J. S. Williams, Au-rich filamentary behavior and associated subband gap optical absorption in hyperdoped Si, Phys. Rev. Mater. 1, 074602 (2017).

[26] Naheed Ferdous and Elif Ertekin, Atomic scale origins of sub-band gap optical absorption in gold-hyperdoped silicon, AIP Adv. 8, 055014 (2018).

[27] R. Curtis Bird and James Stanislaus Williams, Ion Beams for Materials Analysis (Elsevier, Marrickville, Australia, 1990).

[28] N. A. Stolwijk, B. Schuster, and J. Hölzl, Diffusion of gold in silicon studied by means of neutron-activation analysis and spreading-resistance measurements, Appl. Phys. A 33, 133 (1984).
[29] J. Wong-Leung, E. Nygren, and J. S. Williams, Gettering of Au to dislocations and cavities in silicon, Appl. Phys. Lett. 67, 416 (1995).

[30] Travis Wiens, Linear regression with errors in $\mathrm{x}$ and $\mathrm{y}$, Matlab File Exchange (2017).

[31] Ideally, this can be confirmed by performing anneals for shorter durations, but the ramp-down time (it takes approximately $20 \mathrm{~s}$ to ramp down from $500^{\circ} \mathrm{C}$ to $300^{\circ} \mathrm{C}$ ) would be non-negligible in such cases.

[32] S. Coffa, L. Calcagno, S. U. Campisano, G. Calleri, and G. Ferla, Diffusion of ion-implanted gold in p-type silicon, J. Appl. Phys. 64, 6291 (1988).

[33] U. Gösele, W. Frank, and A. Seeger, Mechanism and kinetics of the diffusion of gold in silicon, Appl. Phys. A: Mater. Sci. Process. 23, 361 (1980).

[34] F. C. Frank and D. Turnbull, Mechanism of diffusion of copper in Germanium, Phys. Rev. 104, 617 (1956).

[35] William R. Wilcox and T. J. LaChapelle, Mechanism of gold diffusion into silicon, J. Appl. Phys. 35, 240 (1964).

[36] Daniel Mathiot, Gold, self-, and dopant diffusion in silicon, Phys. Rev. B 45, 13345 (1992).

[37] T. K. Monson, J. A. Van Vechten, Q. S. Zhang, and R. K. Graupner, Comment on "gold, self-, and dopant diffusion in silicon", Phys. Rev. B 49, 2972 (1994).

[38] Peter Pichler, Intrinsic Point Defects, Impurities, and Their Diffusion in Silicon (Springer-Verlag Wien, Vienna, 2004), 1 st ed.

[39] J. S. Williams, M. J. Conway, B. C. Williams, and J. WongLeung, Direct observation of voids in the vacancy excess region of ion bombarded silicon, Appl. Phys. Lett. 78, 2867 (2001).

[40] J. A. Kittl, P. G. Sangers, M. J. Aziz, D. P. Brunco, and M. O. Thompson, Complete experimental test of kinetic models for rapid alloy solidification, Acta Metall. 48, 4797 (2000).

[41] The same $E_{a}$ for both $\mathrm{Au}$ concentrations also implies that the oxide layer, which is known to inject excess interstitials and could enhance the diffusion rate of interstitial-mediated species such as Au [45], does not have a significant effect in our case. Such a result is not unexpected, since the concentration of $\mathrm{Au}$ in the hyperdoped $\mathrm{Si}$ (in the order of a few atomic percent) is presumably much higher than the concentration of interstitials.

[42] Seibt M. Myers, M. Seibt, and W. Schröter, Mechanisms of transition-metal gettering in silicon, J. Appl. Phys. 88, 3795 (2000).

[43] Wenjie Yang, Naheed Ferdous, Peter J. Simpson, Austin J. Akey, Michael J. Aziz, Elif Ertekin, and J. S. Williams, "Vacancy trapping in Si during rapid resolidification from a melt" (to be published).

[44] Such a process is akin to Ostwald ripening and is expected from a thermodynamic point of view. The formation of larger Au clusters reduces the surface-area-to-volume ratio and thus minimizes surface free energy.

[45] S. M. Hu, Formation of stacking faults and enhanced diffusion in the oxidation of silicon, J. Appl. Phys. 45, 1567 (1974). 